\section{Cardiac Indicators, Serum Antioxidant Activity, and Growth Performance as Affected by Hawthorn Extract (Crateagus oxyacantha) in Pulmonary Hypertensive Chickens}

EAuthor(s)

Ahmadipour B' (iD https://orcid.org/0000-0002-4254-145x Kalantar M" (D) https://orcid.org/0000-0003-0183-2151 Kalantar MH"I (iD) https://orcid.org/0000-0001-9273-7584

Department of Animal Science, Shahrekord University, Shahrekord 88186-34141, Iran.

Department of Animal Science, Qom's Agricultural Research and education Center, Agricultural Research, Education, and Extension Organization, Jihad-e-Keshavarzi Ministry, Qom, Iran.

III Student Research Committee, Arak University of Medical Science, Arak, Iran.

\section{nMail Address}

Corresponding author e-mail address Majid Kalantar

Department of Animal Science, Qom's Agricultural Research and education Center, Agricultural Research, Education, and Extension Organization - AREEO, Jihad-e-Keshavarzi Ministry, Qom, Iran P.O Box 165.

Phone: \pm 982536618222

Email: m2332012@gmail.com

\section{EKeywords}

Chicken, Hawthorn flavonoid extract, PHS.

\section{ABSTRACT}

Hawthorn has been traditionally used to improve blood circulation and medicating cardiac disorders as well as blood stasis and elimination of pulmonary hypertension. An experiment was conducted to evaluate the effect of different levels of hawthorn extract in the drinking water on cardiac indicators, serum antioxidant activity, growth and immunity of pulmonary hypertensive broilers reared at high altitude and prone to ascites. A total of 225 one-day-old unsexed broiler chicks (Ross308) were randomly assigned to 15 floor pens. A basal corn-soy based diet was formulated for all treatments. The treatments consisted of the addition of $0,0.1$, or $0.2 \mathrm{~mL}$ of hawthorn extract per liter of drinking water. Both levels of hawthorn flavonoid extract significantly $(p<0.05)$ improved feed intake, body weight gain and FCR, and significantly $(p<0.05)$ reduced abdominal fat, liver, and heart relative weights, as well as the number of birds affected with PHS compared with the controls. RV and TV were significantly $(p<0.05)$ lower in hawthorn flavonoid extract fed birds than the controls in addition of significantly $(p<0.05)$ lower TBARS but higher NO serum levels. In conclusion, hawthorn flavonoid extract may effectively be used as an herbal medicine to prevent pulmonary hypertension and cardiac disorders in chickens.

\section{INTRODUCTION}

Broiler chickens with rapid growth rate are susceptible to pulmonary hypertension syndrome (PHS) due to the imbalance between the oxygen-demanding muscles and the oxygen-supplying organs, such as the heart and the lungs (Ahmadipour et al., 2015). Intensive genetic selection in modern broiler chickens has reduced heart and lung size, increasing their sensitivity to PHS, especially at high altitudes with low oxygen pressure (Khajali \& Fahimi, 2010; Behrooj et al., 2012). Developed pulmonary hypertension and right ventricular failure (RVF) are subsequent effects of hypoxia and vasoconstriction of arterioles, which leads to ascites and PHS (Khajali et al., 2011; Wideman et al., 2013).

Crataegus oxyacantha (Hawthorn) is a member of the Rosaceae family (5 to $10 \mathrm{~m}$ tall shrub) that grows in Asia and most parts of the world (Chang et al., 2002). The fruits, leaves and stems of this medicinal plant are traditionally used for treatment of cardiac diseases and cardiovascular problems, such as heart failure, hypertension with myocardial injuries, angina pectoris, arrhythmia, and as anti-atherosclerotic agent (Salehi et al., 2009), improving blood circulation system, and eliminating blood stasis, hypertension (Chen, 1998; Brixius et al., 2006), and inflammation (Kao et al., 2005; Kao et al., 2007).Flavonoids (1 to $2 \%$ in the fruits, leaves and flowers) and oligomeric proanthocyanidins (OPCs, 1 to $3 \%$ in the fruits or leaves with flowers) are the main chemical components of 
hawthorn (Chang et al., 2002; Kao et al., 2005; Barros et al., 2011; Kirakosyan et al., 2003). However, other bioactive components, such as triterpene acids (Kao et al., 2007), organic acids (Liu 1995; Liu et al., 2011), sterols, and cardio-active amines (Long et al., 2006) are present at different levels in hawthorn extract. Many hawthorn preparations are standardized based on their flavonoid and OPC contents (Hassanpour et al., 2005). The extract of hawthorn fruits, leaves, or flowers have potential antioxidant and free radical scavenging activities due to the presence of epicatechin, hyperoside, and chlorogenic acid (Chang et al., 2002; Barros et al., 2011). These compounds are reported to have many pharmacological actions, including neuroprotective, hepatoprotective, cardioprotective, and nephroprotective effects (Chang et al., 2002; Kirakosyan et al., 2003). Furthermore, the hawthorn fruit has tonic effects on the heart and may reduce cardiovascular risk factors (Long et al., 2006; Salehi et al., 2009).

Some Crataegus oxyacantha compounds have antioxidant activity as well as many positive pharmacological effects. So the objectives of the present study were to examine the effects of different levels of flavonoid extract of Crataegus oxyacantha in the drinking water on the prevention of cardiac disorders, serum antioxidant activity, growth performance of pulmonary hypertensive chickens.

\section{MATERIALS AND METHODS}

\section{Birds and experimental facility}

The experiment was conducted in the experimental facility of Shahrekord University $(2,100 \mathrm{~m}$ altitude), Shahrekord, Iran, to evaluate the effect of different levels of hawthorn extract in the drinking water on the physiological responses of chickens affected with PHS and ascites. According to similar studies (Ahmadipour et al., 2015; Hassanpour et al., 2005; Hassanpour et al., 2008), hypobaric hypoxia was defined as reduced partial oxygen pressure that occurs at altitudes higher than $1500 \mathrm{~m}$. Therefore, comparing to partial oxygen pressure of $21 \%$ at sea level, the partial oxygen pressure in the experimental site calculated as $15.75 \%$ and considered as an hypobaric hypoxia condition that may cause PHS and ascites (Julian 2000; Wideman et al., 2013).

The study was performed in accordance with the recommendations in the Guide for the Care and Use Committee of Shahrekord University, coordinated by $\mathrm{NIH}$ guide for the care and use of laboratory animals, National Research Council Press (2009).
A total of 225 one-day-old unsexed Ross308 broiler chicks were randomly distributed in 15 floor pens measuring $2 \mathrm{~m}^{2}$. Each pen was equipped with a bell drinker and a trough feeder. The average body weights of all pens $(46.8 \pm 1.2 \mathrm{~g})$ were similar at the beginning of the experiment. The temperature of the experimental farm was set at about $32^{\circ} \mathrm{C}$ during week 1 , then reduced at a rate of $3{ }^{\circ} \mathrm{C}$ from week 2 to week 4 , and finally set at $22{ }^{\circ} \mathrm{C}$ until the end of trial. All chicks had free access to feed and water and were provided with $23 \mathrm{~h}$ light and $1 \mathrm{~h}$ dark throughout the trial.

\section{Treatments}

Experimental starter (1-3 weeks of age) and grower (3-6 weeks of age) diets, based on corn and soybean meal, were formulated according to the NRC (1994) recommendations. The treatments consisted the addition of $0.0,0.1$, or $0.2 \mathrm{~mL}$ of hawthorn flavonoid extract per liter of drinking water (equal to 0.0, 0.01, and $0.02 \%$ ). According to the manufacturer, the flavonoid extract of Crataegus oxyacantha contains biologically-active flavonoid compounds (polyphenols) like anthocyanidins and proanthocyanidins (also known as bioflavones or procyanidins). Each $\mathrm{mL}$ of oral hawthorn (Crataegus) flavonoid extract contained 2.5 mg of total flavonoid compounds in form of hyperoside, and was produced by Iran-Darouk Pharmacy Co, under production code of 3067-88-02.

\section{Measurements}

Body weight was measured when birds were 1 and 42 days of age. Feed intake (FI) was measured as gram per bird throughout the 42 days of rearing period. Consequently, the feed conversion ratio (FCR) was calculated for the same period. At the end of trial (42 days of age), 10 birds per treatment were selected for blood sampling and processing.

Blood samples $(3 \mathrm{~mL}$ ) were collected from the brachial vein, and after coagulation, centrifuged at $2500 \mathrm{~g}$ for $10 \mathrm{~min}$ to obtain the serum. Serum samples were used for the determination of nitric oxide (NO) and thiobarbituric acid (TBARS). Serum NO was measured according to the method of Behrooj et al. (2012). Serum TBARS concentration as a biomarker of lipid peroxidation and oxidative stress was assayed by the method described by Antolovich et al. (2002).

For measuring hematocrit, samples of blood were collected in micro-hematocrit tubes. An aliquot of blood was smeared on glass slides for the determination 
of differential leukocyte count using a Neubauer hematocytometer (Fudge, 2000). After staining with May-Grunwald and Giemsa, 100 leukocytes, including granular (heterophils) and non-granular (lymphocytes) were enumerated and the heterophil to lymphocyte ratio $(\mathrm{H}: \mathrm{L})$ was calculated. All chemical reagents were obtained from Sigma-Aldrich Co. (Sigma-Aldrich Co., St. Louis, MO, USA).

After the blood collection, selected birds were sacrificed and body weight, hot carcass weight, and liver, heart and abdominal fat weights were recorded.

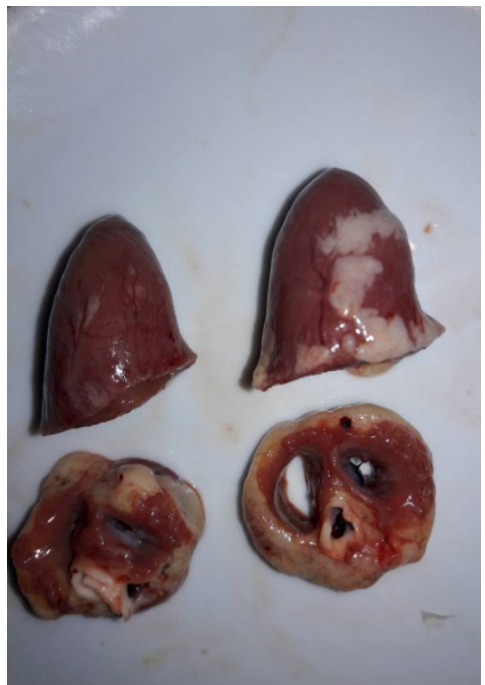

Figure 1 - Normal heart (left) Heart with PHS (right).

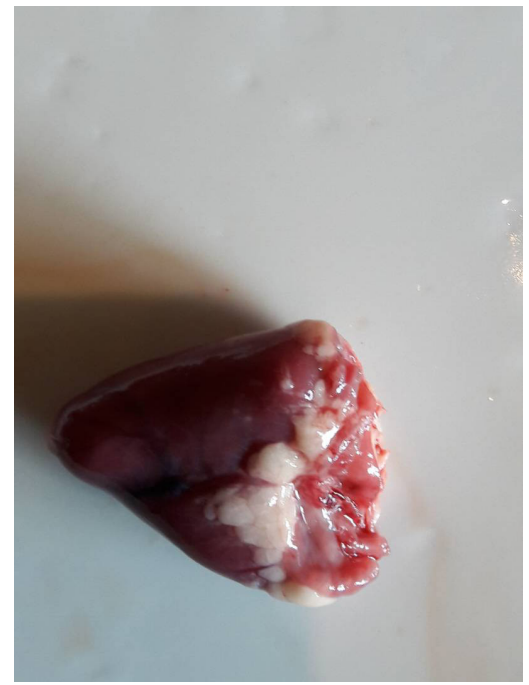

Figure 2 - Enlarged ventricles in aheart with PHS.
Cardiac parameters were measured according to Julian (1987). The heart was removed and stripped of auricles, major vessels and fat. The RV (right ventricle including the valve) was carefully separated from the left ventricle (LV) and septum. The RV was weighed, the left ventricle and septum were added, and finally TV (total ventricles) was weighed. The RV:TV ratio index was also calculated (Fig.1 to 3). The RV:TV ratio is an important index for evaluating pulmonary hypertension. RV:TV ratios greater than 0.25 are considered as pulmonary hypertension (Khajali et al., 2011; Wideman et al., 2013).

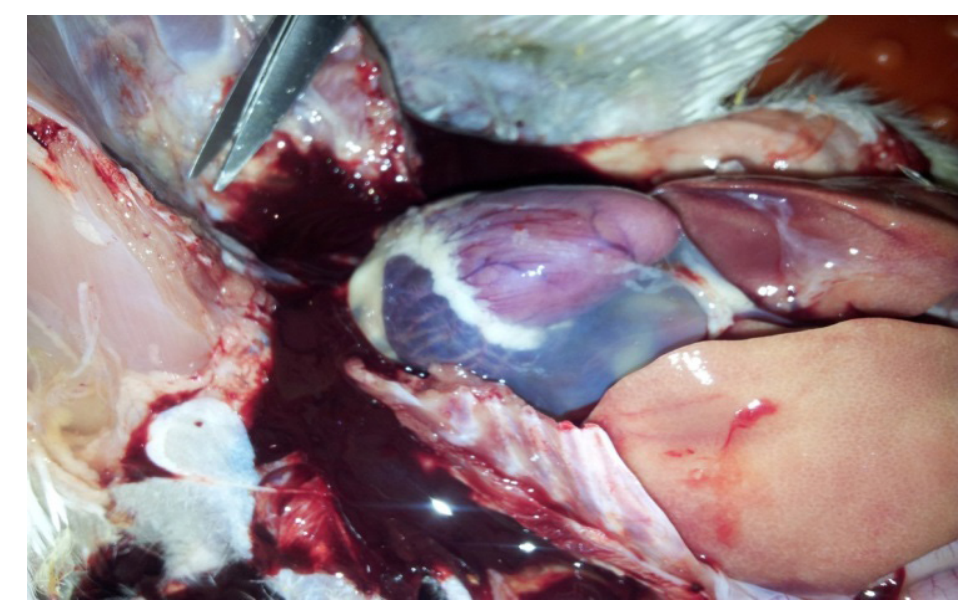

Figure 4 -An example of pulmonary hypertensive chicken with enlarged heart and liquid aggregation in pericardium.

\section{Statistical analysis}

Results were analyzed using the GLM procedure of SAS software (SAS, 2007) according to a completely randomized experimental design. Data were subjected to a nested design when sampling effect within pens was detected. The statistical model used for growth performance data was $Y_{i j}=\mu+T_{i}+e_{i j}$. For other traits with sampling effects, the model was $Y_{i \mathrm{ijk}}=\mu+T_{i}+e_{i j}+\varepsilon_{i j k}$. In these models, $Y_{i j}$ and $Y_{i j k}$ are observations; $\mu$ is the general mean; $\mathrm{Ti}$ is the effect of treatment $i$; $e_{i j}$ is random error; and $\varepsilon_{i j k}$ is subsampling error. Means were separated by Duncan's multiple range test at $p<0.05$. 


\section{RESULTS}

Effects of different hawthorn flavonoid extract levels in the drinking water on broiler growth performance are shown in Table 1. Feed intake, body weight gain and FCR significantly $(p<0.05)$ improved when hawthorn flavonoid extract supplied at 0.1 and $0.2 \mathrm{~mL} / \mathrm{L}$ drinking water throughout the trial.

Table 1 - Effects of hawthorn flavonoid extract on the growth performance of 42-d-old broilers.

\begin{tabular}{lcccc}
\hline \multicolumn{5}{c}{$\begin{array}{c}\text { Hawthorn flavonoid extract levels } \\
\text { (mL/L drinking water) }\end{array}$} \\
\hline Item & Control (0) & 0.1 & 0.2 & SEM \\
\hline Fl (g/bird) & $3887.7^{\mathrm{b}}$ & $4001.6^{\mathrm{a}}$ & $3961.2^{\mathrm{ab}}$ & 55.48 \\
$\mathrm{BWG}(\mathrm{g} / \mathrm{b}$ ird) & $1998.2^{\mathrm{b}}$ & $2186.6^{\mathrm{a}}$ & $2238.2^{\mathrm{a}}$ & 40.47 \\
$\mathrm{FCR}(\mathrm{g} / \mathrm{g})$ & $1.95^{\mathrm{a}}$ & $1.83^{\mathrm{b}}$ & $1.77^{\mathrm{c}}$ & 0.03 \\
\hline a,bSuperscripts in the same row with different letters are statistically different $(p<0.05)$. \\
Fl=Feed Intake; BWG= Body Weight Gain; FCR=Feed Conversion Ratio.
\end{tabular}

Table 2 shows the carcass trait results of 42-d-old broilers supplied with different levels of hawthornflavonoid extract in the drinking water. Broilers consuming both hawthornflavonoid extract levels in the drinking water ( 0.1 and $0.2 \mathrm{~mL} / \mathrm{L}$ ) had higher body weight at slaughter than those of the control group $(p<0.05)$. The inclusion of hawthornflavonoid extract in the drinking water significantly $(p<0.05)$ reduced abdominal fat, liver and heart weights compared with the control birds. In addition, both hawthornflavonoid extract levels ( 0.1 and $0.2 \mathrm{~mL}$ ) per $L$ of drinking water significantly $(p<0.05)$ reduced the number of birds affected with PHS.

Table 2 - Effect of hawthorn flavonoid extract on the carcass traits of 42 -d-old broilers and on the number of birds affected with PHS ${ }^{1}$

\begin{tabular}{lcccc}
\hline \multicolumn{4}{c}{$\begin{array}{c}\text { Hawthornflavonoid extract levels } \\
(\mathrm{mL} / \mathrm{L} \text { drinking water) }\end{array}$} \\
\hline Item (gram) & Control (0) & 0.1 & 0.2 & SEM \\
\hline Body weight at slaughter & $2101.3^{\mathrm{b}}$ & $2152.5^{\mathrm{a}}$ & $2260.1^{\mathrm{a}}$ & 44.48 \\
Hot carcass weight & $1635.5^{\mathrm{c}}$ & $1737.8^{\mathrm{b}}$ & $1816.6^{\mathrm{a}}$ & 27.87 \\
Abdominal fat & $22.7^{\mathrm{a}}$ & $19.9^{\mathrm{b}}$ & $19.1^{\mathrm{b}}$ & 0.76 \\
Liver & $47.2^{\mathrm{a}}$ & $43.8^{\mathrm{b}}$ & $41.1^{\mathrm{c}}$ & 1.03 \\
Heart & $13.5^{\mathrm{a}}$ & $12.3^{\mathrm{b}}$ & $11.5^{\mathrm{c}}$ & 0.29 \\
Affected birds with & $57.34^{\mathrm{a}}$ & $44.66^{\mathrm{b}}$ & $36.84^{\mathrm{c}}$ & 3.85 \\
PHS $(\%)$ & & & & \\
\hline
\end{tabular}

${ }^{1}$ PHS: Pulmonary Hypertension Syndrome. Each mean represents values from 10 replicates.

a,bSuperscripts in the same row with different letters are statistically different $(P<0.05)$.

Table 3 shows the blood parameters of broilers receiving different hawthorn flavonoid extract levels in the drinking water. Chickens receiving hawthorn flavonoid extract at 0.1 and $0.2 \mathrm{~mL} / \mathrm{L}$ drinking water showed significantly lower $(p<0.05)$ heterophil percentage, but significantly higher $\quad(p<0.05)$ lymphocyte percentage than that of the control group. In addition, significant reductions $(p<0.05)$ in heterophil to lymphocyte ratio and hematocrit were observed in birds fed hawthorn flavonoid extract compared with the control birds.

Table 3 - Effects of hawthorn flavonoid extract on blood immune cells and hematocrit ${ }^{1}(\%)$ of 42 -d-old broilers.

\begin{tabular}{lcccc}
\hline Item & $\mathrm{Htr}$ & Lym & $\mathrm{H} / \mathrm{L}$ & $\mathrm{HCT}$ \\
\hline Control(0) & $40.88^{\mathrm{a}}$ & $46.13^{\mathrm{b}}$ & $0.89^{\mathrm{a}}$ & $39.75^{\mathrm{a}}$ \\
0.1 & $36.14^{\mathrm{b}}$ & $52.57^{\mathrm{a}}$ & $0.69^{\mathrm{b}}$ & $36.13^{\mathrm{b}}$ \\
0.2 & $33.50^{\mathrm{b}}$ & $55.38^{\mathrm{a}}$ & $0.61^{\mathrm{b}}$ & $32.50^{\mathrm{c}}$ \\
SEM & 1.75 & 2.52 & 0.16 & 1.65 \\
\hline
\end{tabular}

'Each value represents the mean of 12 replicates; $\mathrm{Htr}=$ Heterophiles: Lym=Lymphocytes; $\mathrm{H} / \mathrm{L}=$ Heterophile to Lymphocyte ratio. $\mathrm{HCT}=$ Hematocrit.

a,bSuperscripts in the same column with different letters are statistically different $(p<0.05)$.

Table 4 shows cardiac indicators of broilers receiving different hawthorn flavonoid extract levels in the drinking water. Hawthorn flavonoid extract at both 0.1 and $0.2 \mathrm{~mL} / \mathrm{L}$ promoted significantly lower $(p<0.05)$ right ventricle (RV) and total ventricle (TV) weights compared with the control group.

Table 4 - Effects of hawthorn flavonoid extract on cardiac indicators ${ }^{1}$ of 42 -d-old broilers (g).

\begin{tabular}{lccc}
\hline Item & RV & TV & RV:TV \\
\hline Control (0) & $3.19^{\mathrm{a}}$ & $9.99^{\mathrm{a}}$ & $0.32^{\mathrm{a}}$ \\
0.1 & $2.20^{\mathrm{b}}$ & $8.99^{\mathrm{b}}$ & $0.25^{\mathrm{b}}$ \\
0.2 & $1.91^{\mathrm{b}}$ & $8.77^{\mathrm{b}}$ & $0.22^{\mathrm{b}}$ \\
SEM & 0.23 & 0.35 & 0.02 \\
\hline
\end{tabular}

'Each value represents the mean of 12 replicates. RV=Right Ventricle; TV=Total Ventricle.

a,bSuperscripts in the same column with different letters are statistically different $(p<0.05)$.

The serum antioxidant activity of broilers receiving different hawthorn flavonoid extract levels in the drinking water is presented in Table 5 . Broilers receiving hawthorn flavonoid extract at level of 0.1 and $0.2 \mathrm{~mL} / \mathrm{L}$ drinking water had significantly $(p<0.05)$ lower serum thiobarbituric acid (TBARS) levels, but higher $(p<0.05)$ nitric oxide (NO) levels than those of the control group.

Table 5 - Effects of hawthorn flavonoid extract on serum antioxidant activity ${ }^{1}$ of 42 -d-old broilers ( $\mathrm{mmol} / \mathrm{L}$ ).

\begin{tabular}{lll}
\hline Item & TBARS & NO \\
\hline Control (0) & $2.09^{\mathrm{a}}$ & $5.32^{\mathrm{c}}$ \\
0.1 & $1.06^{\mathrm{b}}$ & $6.71^{\mathrm{b}}$ \\
0.2 & $0.84^{\mathrm{c}}$ & $8.06^{\mathrm{a}}$ \\
SEM & 0.11 & 0.36 \\
\hline
\end{tabular}

'Each mean represents values from 12 replicates. TBARS=Thiobarbituric Acid. $\mathrm{NO}=$ Nitric Oxide.

a,bSuperscripts in the same column with different letters are statistically different $(p<0.05)$. 


\section{DISCUSSION}

The findings of this study show that the significant improvements in growth traits, including feed intake, body weight gain and feed conversion ratio obtained when broiler were offered 0.1 and $0.2 \mathrm{~mL}$ hawthorn flavonoid extract/L drinking water may be attributed to their better health status. The observed improvements in carcass traits, as well as lower abdominal fat and liver and heart weights may be due to the effects of naturally-occurring polyphenols (flavonoids) and oligomeric proanthocyanidins (OPCs) in hawthorn (Crateagusoxyacantha) (Mladěnka et al., 2010). Polyphenols, including flavonoids and non-flavonoids compounds, exhibit a wide range of beneficial pharmaceutical effects, such as growth-promoting, anti-oxidative, sedative, antibacterial and anti-viral actions (Barros et al., 2011; Surai, 2014).

The lower percentage of birds affected with pulmonary hypertensive in the groups that received 0.1 and $0.2 \mathrm{~mL}$ hawthorn flavonoid extract/L drinking water may be explained by the pharmacological effects of the flavonoid extract, in particular by the presence of bioactive components such as epicatechin, hyperoside, and chlorogenic acid, which have potent antioxidant and free radical scavenging activities that counteract the oxidative damages caused by pulmonary hypertension or other cardiovascular lesions (Chang et al., 2002; Barros et al., 2011).

According to the results presented in Table 3, a significant improvement was observed in hematological parameters of broilers receiving0.1 and $0.2 \mathrm{~mL}$ hawthorn flavonoid extract in the drinking water, as shown by their lower percentage of heterophiles, but higher percentage of lymphocyte compared with the control group, which resulted in a reduction of the heterophil to lymphocyte ratio. Both evaluated hawthorn flavonoid extract levels reduced HCT\%, which means lower red blood cell concentration and, consequently, blood viscosity. Hypoxemia stimulates erythropoiesis, increasing the hematocrit and blood $\mathrm{O}_{2}$ carrying capacity. Significant increases in the hematocrit and the reduced deformability of immature erythrocytes may potentially increase blood viscosity, and thereby increasing the resistance to blood flow (Hamal et al., 2012).

The significant reductions in the $\mathrm{H}: \mathrm{L}$ ratio and $\mathrm{HCT} \%$ recorded in the broilers receiving hawthorn flavonoid extract are consistent with the reduced oxidative stress (lower TBARS values, related with lipid oxidation rate). The $\mathrm{H}: \mathrm{L}$ ratio is widely accepted as a reliable and accurate physiological indicator of the stress response in chickens is an index used to describe stress status in the chicken (Khajali et al., 2011; Ahamadipour et al., 2015). Differential leukocyte counts show that the normal heterophil:lymphocyte ratio is about 1:2; however, this ratio increases when birds are submitted to stress conditions by increasing the number of circulating heterophils (Gross \& Siegel 1983). Therefore, the results of the present study suggest that hawthorn flavonoid extract suppressed ROS production and reduced oxidative stress, as shown by the reduced TBARS levels and H:L ratios, which ultimately led to better live performance.

As a biological rule, RV:TV values greater than 0.25 indicate pulmonary hypertension (Wideman et al., 2013; Ahamadipour et al., 2015). In the control group, the mean RV:TV value was 0.32 and greater than other groups, which implies that a greater number of birds in this group experienced pulmonary hypertension.

The higher serum $\mathrm{NO}$ levels determined in the groups receiving 0.1 and $0.2 \mathrm{~mL}$ hawthorn flavonoid extract is possibly a result of the overproduction of NO in the heart induced by bioactive hawthorn flavonoid extract compounds. It has been demonstrated that, under normal conditions, NO production in the myocardiocytes is also normal. Though NO is an important regulator of cardiac function due to its role in the control of myocardial energetics, myocardial regeneration, hypertrophic remodeling, and improvement of ventricular diastolic distensibility (Ahamadipour et al., 2015). It was reported that impaired NO synthesis in the heart ventricles is involved in the pathophysiology of cardiac failure in chickens with pulmonary hypertension (Hassanpour et al., 2009).

Based on the results shown in Table 5, the consumption o fhawthorn flavonoid extract at levels of 0.1 and $0.2 \mathrm{~mL}$ per liter of drinking water significantly reduced TBARS levels, which is an important indicator of oxidative stress related to body lipid oxidation. It is obvious that chickens are very susceptible to oxidative stress due to their intensive metabolic rate, high blood sugar concentration, and high body temperature, resulting which resulted in high production of ROS (Ahamadipour et al., 2015). Therefore, antioxidant potency is crucial to chickens body. Some of bioactive compounds in hawthorn flavonoid extract such as flavonoids and particularly OPCs contribute to the protection against oxidative stress and lipid peroxidation, along with an increase the activities of other serum antioxidant enzymes such as glutathione 
peroxidase, superoxide dismutase and catalase, which counteract the oxidative stress (Chang et al., 2002; Barros et al., 2011).

In this study abdominal fat deposition and liver relative weight were significantly reduced in broilers consuming different levels of hawthorn flavonoid extract. Hawthorn flavonoid extract has lipolytic effects, which are attributed to flavonoids and OPCs as well as phenolic compounds (Rajendran et al., 1996; Zhang et al., 2002). Lipid-lowering effects of bioactive compounds such as flavonoids have been well documented (Anila \& Vijayalakshmi, 2002; Chen $\& \mathrm{Li}, 2007)$. The lower liver relative weight observed in broilers consuming different levels of hawthorn flavonoid extract is consistent with the lower lipogenesis rate, as demonstrated by the reduced abdominal fat percentage. In chickens, the liver is the major lipogenesis organ (Behrooj et al., 2012), and reduced liver weight or size reflects lower lipogenesis, as observed in the present study in the birds consuming hawthorn flavonoid extract in the drinking water.

Lower RV:TV ratio was detected in the birds consuming hawthorn flavonoid extract compared with the control birds. These results confirm the ability of hawthorn flavonoid extract to prevent heart hypertrophy and right ventricular hypertrophy in particular. As shown in Table 4, the RV:TV ratio higher than 0.25 in the birds of the control group indicate that they were in pre-ascitic condition and this situation was improved when the birds consumed hawthorn flavonoid extract at levels of 0.1 and 0.2 $\mathrm{mL} / \mathrm{L}$ drinking water. In this regard, a significant decline in the number of birds affected with PHS was observed when hawthorn flavonoid extract was offered in the drinking water relative to the control group.

In addition, in the present study, serum TBARS levels declined while NO levels increased in receiving hawthorn flavonoid extract compared with the controls. Research has shown that the overproduction of NO along with increasing of serum antioxidant activity reduces hypertension, and promotes endothelium-dependent relaxation in different models of hypertension (Ahamadipour et al., 2015; Hassanpour et al., 2009). This finding explains significant reduction in the incidence of PHS in the birds consuming0.1 and $0.2 \mathrm{~mL}$ hawthorn flavonoid extract/L drinking water. According to relevant reports, the vascular remodeling of lung vessel beds contributes to the mortality of broilers with pulmonary hypertension syndrome (Khajali et al., 2011; Wideman et al., 2013). The flavonoid content of hawthorn extract prevents the proliferation of vascular smooth muscle cells, and inhibits thickening of the intima and narrowing of the vessels, as well as exerts considerable collagen stabilizing effect (Chen et al., 1998; Brixius et al., 2006; Lu et al., 2006). Therefore, flavonoids and OPCs are effective agents in preventing cardiovascular diseases (Lu et al., 2006; Salehi et al., 2009). Flavonoids and OPCs have also vasorelaxant potential and considering the fact that flavonoids and OPCs are the main active components of hawthorn (Crateagusoxyacantha), this medicinal plant could effectively prevent PHS in broiler chickens.

\section{CONCLUSION}

The results of this study have shown that hawthorn flavonoid extract levels of 0.1 and $0.2 \mathrm{~mL} /$ Lof drinking water significantly improved growth and carcass characteristics. In addition, especially at the level of 0.2 $\mathrm{mL} / \mathrm{L}$, hawthorn flavonoid extract was able to ameliorate cardiac problems and prevent pulmonary hypertension in chickens. Higher serum concentration of $\mathrm{NO}$ and lower serum concentration of TBARS, together with modulation of $\mathrm{HCT} \%$ and $\mathrm{H} / \mathrm{L}$ ratio were the other important positive effects of hawthornflavonoid extract. Therefore, hawthornflavonoid extract is an effective herbal medicine that may be used for the prevention of pulmonary hypertension and cardiac disorders in chickens.

\section{ACKNOWLEDGMENTS}

This research was funded by Shahrekord University (fund number 141-56), and Iran Ministry of Science, Research, and Technology (MSRT). Authors thanks for technical supports of Animal Science Department of Agricultural Research Center of Qom (QARC), Qom, Iran.

\section{REFERENCES}

Ahmadipour B, Hassanpour H, Asadi E, Khajali F, Rafiei F, Khajali F. Kelussia odoratissima Mozzaf- A promising medicinal herb to prevent pulmonary hypertension in broiler chickens reared at high altitude. Journal of Ethnopharmacology 2015;159(1):49-54.

Anila L, Vijayalakshmi NR. Flavonoids from Emblica officinalis and Mangifera indica effectiveness for dyslipidemia. Journal of Ethnopharmacology $2002 ; 79(1): 81-87$

Antolovich M, Prenzler PD, Patsalides E, McDonald S, Robards K. Methods for testing antioxidant activity. Analyst 2002;127:183-198.

Barros L, Carvalho AM, Ferreira IC. Comparing the composition and bioactivity of Crataegus monogyna flowers and fruits used in folk medicine. Phytochemical Analysis 2011;22(2):181-188. 
Behrooj N, Khajali F, Hassanpour H. Feeding reduced-protein diets to broilers subjected to hypobaric hypoxia is associated with the development of pulmonary hypertension syndrome. British Poultry Science 2012;53(5):658-664

Brixius K, Willms S, Napp A, Tossios P, Ladage D, Bloch W, et al. Crataegus special extract WS 1442 induces an endothelium-dependent, NOmediated vasorelaxation via eNOS-phosphorylation at serine 1177. Cardiovascular Drugs and Therapy 2006;20(3):177-184.

Chang Q, Zuo Z, Harrison F, Chow MSS. Hawthorn. Journal of Clinical Pharmacology 2002;42(6):605-612.

Chen ZY, Zhang ZS, Kwan KY, Zhu M, Ho W, Huang Y. Endotheliumdependent relaxation induced by hawthorn extract in rat mesenteric artery. Life Sciences 1998;63(22):1983-1991.

Chen J, Li X. Hypolipidemic effect of flavonoids from mulberry leaves in triton WR-1339 induced hyperlipidemic mice. Journal of Asian Pacific Clinical Nutrition 2007;16(Suppl 1):290-294

Fudge AM. Laboratory Medicine: avian and exotic pets. Philadelphia: Saunders; 2000.

Gross WB, Siegel HS. Evaluation of the heterophil/ lymphocyte ratio as a measure of stress in chickens. Avian Disease 1983;27:972-979.

Hamal KR, Erf GF, Anthony NB, Wideman RF. Immunohistochemical examination of plexiform-like complex vascular lesions in the lungs of broiler chickens selected for susceptibility to pulmonary arterial hypertension. Journal of Avian Pathology. 2012;41:211-219.

Hassanpour H, Teshfam M, Modirsanei M, Emadi L. Comparative studies of Electrocardiographic parameters, mean electrical axes and cardiac index in two groups of normal and experimentally ascitic broilers. Journal of Veterinary Research 2005;60(12):333-337.

Hassanpour H, Zamanimoghadam AK, Teshfam M, Zarei H. Effect of ascorbic acid on the electrocardiogram of broiler chickens raised at high altitude. Nigerian Veterinary Journal 2008;29(2):8-14.

Hassanpour H, Yazdani A, Khabir Soreshjani K, Asgharzadeh S. Evaluation of endothelial and inducible nitric oxide synthase genes expression in the heart of broiler chickens with experimental pulmonary hypertension. British Poultry Science 2009;50(6):725-732.

Julian RJ. The effect of increased sodium in the drinking water on right ventricular hypertrophy, right ventricular failure and ascites on broiler chickens. Avian Pathology 1987;16(1):61-71.

Julian RJ. Physiological, management and environmental triggers of the ascites syndrome: a review. Avian Pathology 2000;29(6):519-527.

Kao E, Wang C, Lin W, Yin Y, Wang C, Tseng TH. Anti-inflammatory potential of flavonoid contents from dried fruit of Crataegus pinnatifidain vitro and in vivo. Journal of Agricultural and Food Chemistry 2005;53(2):430-436.

Kao ES, Wang CJ, Lin WL, Chu CY, Tseng TH. Effects of polyphenols derived from fruit of Crataegus pinnatifida on cell transformation, dermal edema and skin tumor formation by phorbol ester application. Food and Chemical Toxicology 2007;45(10):1795-1804.
Khajali F, Fahimi S. Influence of dietary fat source and supplementary $\alpha$-tocopheryl acetate on pulmonary hypertension and lipid peroxidation in broilers. Journal of Animal Physiology and Animal Nutrition 2010;94(4):767-772.

Khajali F, Liyanage R, Wideman RF. Methylglyoxal and pulmonary hypertension in broiler chickens. Poultry Science 2011;90(6):12871294.

Kirakosyan A, Seymour E, Kaufman PB, Warber S, Bolling S, Chang SC. Antioxidant capacity of polyphenolic extracts from leaves of Crataegus laevigata and Crataegusmonogyna (Hawthorn) subjected to drought and cold stress. Journal of Agricultural and Food Chemistry 2003;51(14):3973-3976.

Liu J. Pharmacology of oleanolic acid and ursolic acid. Journal of Ethnopharmacology. 1995;49(2):57-68

Liu P, Yang B, Kallio H. Phenolic Compounds in hawthorn (Crataegusgrayana) fruits and leaves and changes during fruit ripening. Journal of Agricultural and Food Chemistry 2011;59(20):11141-11149.

Long SR, Carey RA, Crofoot KM, Proteau PJ, Filtz TM. Effect of hawthorn (Crataegusoxycantha) crude extract and chromatographic fractions on multiple activities in a cultured cardiomyocyte assay. Phytomedicine 2006;13(9-10):643-650

Lu Q, Qiu TQ, Yang H. Ligustilide inhibits vascular smooth muscle cells proliferation. European Journal of Pharmacology 2006;542(1-3):136140 .

Mladěnka P, Zatloukalová L, Filipský T, Hrdina R. Cardiovascular effects of flavonoids are not caused only by direct antioxidant activity. Free Radical Biology and Medicine 2010;49(6):963- 975.

NRC - National Research Council Press. NIH guide for the care and use of laboratory animals. $8^{\text {th }}$ ed. Washington; 2009.

NRC - National Research Council Press. Nutrient requirements for poultry. $9^{\text {th }}$ ed. Washington; 1994

Rajendran S, Deepalakshmi PD, Parasakthy K, Devaraj H, Devaraj SN Effect of tincture of Crataegus on the LDL-receptor activity of hepatic plasma membrane of rats fed an atherogenic diet. Atherosclerosis 1996;123(1-2):235-241.

SAS Institute. SAS/STAT user's guide. Release 9. Cary; 2007.

Salehi S, Long SR, Proteau PJ, Filtz TM. Hawthorn (Crataegusmonogyna Jacq.) extract exhibits atropine-sensitive activity in a cultured cardiomyocyte assay. Journal of Natural Medicines 2009;63(1):1-8.

Surai PF. Polyphenol compounds in the chicken/animal diet:from the past to the future. Journal of Animal Physiology and Animal Nutrition 2014;98(1):19-31.

Zhang Z, Ho WK, Huang Y, James AE, Lam LW, Chen ZY. Hawthorn fruit is hypolipidemic in rabbits fed a high cholesterol diet. Journal of Nutrition 2002;132(1):5-10.

Wideman RF, Rhoads DD, Erf GF, Anthony NB. Pulmonary arterial hypertension (PAH, ascites syndrome) in broilers:a review. Poultry Science 2013;92(1):64-83. 
